\title{
Uso da Soma Cumulativa dos Desvios para Avaliação da Proficiência no Ensino do Bloqueio Subaracnóideo *
}

\section{Cumulative Sum Analysis Applied to the Teaching of Spinal Anesthesia}

Getúlio Rodrigues de Oliveira Filho, $T S A^{1}$, Sérgio Galluf Pederneiras, $T S A^{2}$, Jorge Hamilton Soares Garcia, TSA ${ }^{3}$

\author{
RESUMO \\ Oliveira Filho GR, Pederneiras SG, Garcia JHS - Uso da Soma \\ Cumulativa dos Desvios para Avaliação da Proficiência no \\ Ensino do Bloqueio Subaracnóideo
}

\begin{abstract}
Justificativa e Objetivos - Medidas objetivas de desempenho são necessárias para o ensino de técnicas anestésicas. Este estudo teve por objetivos aplicar e descrever o método da soma cumulativa dos desvios para a construção de curvas de aprendizado da punção subaracnóidea.

Método - O sucesso da punção subaracnóidea na primeira tentativa, no primeiro espaço abordado, e o sucesso da anestesia de 275 bloqueios subaracnóideos realizados por médicos em especialização (ME) durante os primeiros seis meses de treinamento foram utilizados para a construção de curvas de aprendizado, utilizando o método das somas cumulativas de desvios. As taxas aceitáveis de falha foram derivadas de uma amostra de 264 bloqueios subaracnóideos realizados por anestesiologistas. O número necessário de bloqueios para se obter proficiência foi calculado, para cada $M E$, para cada atributo.
\end{abstract}

Resultados - Houve uma grande variabilidade em relação ao número de bloqueios necessários para se atingir a proficiência, dependendo do atributo e do ME. Contudo, a maioria dos ME atingiu proficiência para os atributos de sucesso durante a primeira tentativa ou no primeiro espaço inter-espinhoso abordado, após 50 bloqueios. Uma taxa de sucesso da anestesia de $90 \%$ foi obtida por todos os ME após 30 bloqueios. Conclusões - O método da soma cumulativa de desvios pode ser utilizado para medir objetivamente o desempenho de médicos em especialização, durante a fase de aprendizado de anestesia subaracnóidea. Um número mínimo de 50 bloqueios é necessário para se obter proficiência em identificar o espaço subaracnóideo.

UNITERMOS - ANESTESIOLOGIA: ensino; TÉCNICAS ANESTÉSICAS, Regional: subaracnóidea

\footnotetext{
* Recebido do (Received from) CET/SBA Integrado de Anestesiologia da SES-SC, Florianópolis, SC

1. Responsável pelo CET/SBA

2. Instrutor Co-responsável pelo CET/SBA

3. Instrutor do CET/SBA
}

Apresentado (Submitted) em 11 de setembro de 2000

Aceito (Accepted) para publicação 23 de novembro de 2000

Correspondência para (Mail to):

Dr. Getúlio Rodrigues de Oliveira Filho

Rua José Cândido Silva 179/402

88075-250 - Florianópolis, SC

E-mail: grof@th.com.br

(c) Sociedade Brasileira de Anestesiologia, 2001

\author{
SUMMARY \\ Oliveira Filho GR, Pederneiras SG, Garcia JHS - Cumulative \\ Sum Analysis Applied to the Teaching of Spinal Anesthesia
}

Background and Objectives - Objective performance measurements are necessary during the learning process of anesthetic techniques. This study aimed at describing the cusum (cumulative sum) analysis to build subarachnoid puncture learning curves.

Methods - The successful subarachnoid space identification during the first attempt, in the first interspace approached, and the success of 275 spinal blocks performed by 5 residents during the initial six-month training period were used to build learning curves through the cusum analysis. Acceptable failure rates were taken from a sample of 264 spinal blocks performed by experienced anesthesiologists. The number of blocks needed for proficiency was calculated for each resident, for each attribute.

Results - A wide variability in the number of blocks needed for attaining proficiency was observed, depending on the resident or on the attribute. However, most residents attained proficiency after 50 blocks. All residents achieved a $90 \%$ success rate after 30 blocks.

Conclusions - Cusum analysis may be used for objectively measuring performance during the learning phase of spinal anesthesia. A minimum of 50 blocks is required in order to attain proficiency in identifying subarachnoid space.

KEY WORDS - ANESTHESIOLOGY: teaching; ANESTHETIC TECHNIQUES, Regional: spinal block

\section{INTRODUÇÃO}

$\mathrm{O}$ aprendizado do bloqueio subaracnóideo começa pelo domínio das técnicas de punção lombar. O ensino inicia-se com supervisão intensiva que diminui progressivamente, à medida que o médico em especialização (ME) vai adquirindo melhor desempenho, até tornar-se dispensável. O ponto de transição entre a supervisão intensiva e a dispensável precisa ser definido de forma objetiva, através de instrumentos de avaliação quantitativa do desempenho, ou seja, do nível de proficiência.

O instrumento de avaliação ideal deve ser de fácil computação e prover dados para o acompanhamento da evolução de cada ME, identificando pontos de desempenho fora dos padrões aceitos e medindo o resultado das intervenções corretivas.

A soma cumulativa dos desvios (SCD) é um dos métodos estatísticos utilizados para controle de qualidade na indústria e já foi utilizada para medir a proficiência de médicos residentes durante o aprendizado de procedimentos como intubação traqueal, anestesias subaracnóidea e peridural e cateterismos venoso central e arterial ${ }^{1}$. Vol. 51, Nº 3, Maio - Junho, 2001 
O método é de fácil computação, necessitando do cálculo de três parâmetros: o desvio sigma ( $s$ ), o limite superior de controle (LSC), calculado a partir de uma taxa inaceitável de faIhas $\left(p_{0}\right)$ e o limite inferior de controle (LIC), calculado a partir de uma taxa aceitável de falhas $\left(p_{1}\right)$. Para o cálculo da SCD, inicia-se com um ponto zero, que representa o primeiro procedimento. A cada sucesso obtido durante a realização de um procedimento subseqüente, subtrai-se da SCD precedente o valor de s e a cada falha, adiciona-se o valor de 1 - $s$. Assim, obtém-se uma curva que representa o aprendizado do procedimento, a qual ascende quando ocorrem falhas e descende, quando ocorrem sucessos. Quando a linha da SCD ultrapassa, a partir de baixo, o LSC, o desempenho é considerado inadequado. Enquanto a linha da SCD está entre LIC e LSC nenhuma decisão estatística pode ser tomada e observações adicionais precisam ser realizadas. Quando a linha da SCD ultrapassa o LIC, a partir de cima, o desempenho é considerado aceitável, por não diferir significativamente de $p_{1}{ }^{2}$.

Assim, este estudo teve como objetivo a construção de curvas de aprendizado de técnicas de punção subaracnóidea, utilizando o método da soma cumulativa dos desvios para determinação do número mínimo de procedimentos que devem ser realizados pelos médicos em especialização (ME) até a obtenção de proficiência.

\section{MÉTODO}

Este estudo obteve aprovação da Comissão de Ética Médica do Hospital Governador Celso Ramos. Foram coletados, prospectivamente, os dados referentes aos primeiros 275 bloqueios subaracnóideos realizados por 5 médicos em especialização, durante os primeiros seis meses de residência (Grupo R). Os ME foram designados A, B, C, D e E. No mesmo período foi constituído um grupo controle (Grupo A) com uma amostra de 264 bloqueios subaracnóideos realizados por 22 anestesiologistas do CET.

No grupo R, os bloqueios foram realizados sob supervisão de instrutores credenciados pela Sociedade Brasileira de Anestesiologia. No tocante à punção lombar, os ME receberam orientação quanto ao posicionamento dos pacientes, à técnica de anti-sepsia e colocação de campos esterilizados, à escolha do espaço inter-espinhoso para punção, à anestesia local, ao direcionamento da agulha e à identificação do espaço subaracnóideo por obtenção de líquido cefalorraquidiano.

Todos os bloqueios foram realizados com agulhas descartáveis, tipo Quincke. De todos os pacientes foram registrados a idade, o sexo, a qualidade da palpação dos processos espinhosos (fácil, difícil ou impossível), a via de acesso ao espaço subaracnóideo (mediano ou paramediano), o decúbito durante a punção lombar (lateral ou sentado), as condições do posicionamento (satisfatório ou insatisfatório) e o calibre da agulha de punção lombar.

Os seguintes parâmetros foram registrados e codificados como 1 , se presente e 0 , se ausente:
1. Atributo 1: sucesso na primeira tentativa - localização do espaço subaracnóideo com apenas uma punção da pele.

2. Atributo 2: sucesso no primeiro espaço - localização do espaço subaracnóideo no primeiro espaço inter-espinhoso abordado, ainda que com mais de uma punção da pele.

3. Atributo 3: sucesso da anestesia - obtenção de anestesia cirúrgica completa.

Para definir os limites de controle para os atributos 1,2 e 3 , foram derivadas as seguintes taxas, de uma amostra de 264 bloqueios subaracnóideos realizados por 22 anestesiologistas do CET, atuando em dois hospitais, durante o mesmo período (Grupo $A)$ :

1. Taxa de sucesso na primeira tentativa - número de bloqueios em que o espaço subaracnóideo foi localizado com apenas uma punção da pele dividido pelo número total de punções multiplicado por 100.

2. Taxa de sucesso no primeiro espaço - número de bloqueios em que o espaço subaracnóideo foi localizado por uma ou mais punções da pele, mas no primeiro espaço inter-espinhoso abordado, dividido pelo número total de bloqueios e multiplicado por 100 .

3. Taxa de sucesso da anestesia - número de bloqueios em que o procedimento cirúrgico foi realizado sem necessidade de complementação do bloqueio por opióides ou anestesia geral, dividido pelo número total de bloqueios e multiplicado por 100 .

Para o atributo $1, p_{1}$ foi estabelecida em 0,35 , por ser de $35 \%$ a taxa de falhas na primeira tentativa do grupo Ae $p_{0}$ foi definida arbitrariamente como 0,5 . Da mesma forma, $p_{0}$ foi estabelecida em 0,1 e $p_{1}$ em 0,2, para o atributo 2. Para o atributo 3, a taxa de insucesso do grupo $A$ foi de $5 \%$, resultando em $p_{0}=$ 0,05 e $p_{1}$ foi definida arbitrariamente em 0,2 . Para o atributo 3 foi utilizada também uma taxa de sucesso de $90 \%$, definida por consenso de especialistas ${ }^{1}$, resultando em $p_{0}=0,1$ e $p_{1}=$ 0,2 . Os valores de $\alpha$ (erro tipo I) e $\beta$ (erro tipo II) foram fixados em 0,1 .

O cálculo das variáveis para a SCD foi feito pelas seguintes fórmulas:

$$
\begin{aligned}
& a=\ln [(1-\beta) / \alpha] \\
& b=\ln [(1-\alpha) / \beta] \\
& P=\ln \left(p_{1} / p_{0}\right) \\
& Q=\ln \left[\left(1-p_{1}\right) /\left(1-p_{0}\right)\right] \\
& \mathrm{LIC}=-\mathrm{b} /(P+Q) \\
& \mathrm{LSC}=\mathrm{a} /(\mathrm{P}+\mathrm{Q}) \\
& s=\mathrm{Q} /(\mathrm{Q}+\mathrm{P})
\end{aligned}
$$

Para cada um dos atributos, foram calculadas as curvas de aprendizado de cada ME, baseadas na soma cumulativa dos desvios. A proficiência em relação a determinado atributo (aceitação da hipótese nula) foi identificada por valores da 
SCD permanentemente inferiores ao LIC. O número de bloqueios até a obtenção de proficiência foram comparados, entre os ME, pelo teste do qui-quadrado e pelo teste exato de Fisher.

Os dados demográficos dos pacientes e as características técnicas dos procedimentos foram comparados, entre os grupos, pelos testes $t$ de Student (variáveis contínuas) ou qui-quadrado (variáveis categóricas).

\section{RESULTADOS}

Os grupos A e R não diferiram quanto à idade. Houve predomínio de pacientes do sexo masculino no grupo $R$. No grupo A, o acesso paramediano e a posição sentada foram utilizados mais freqüentemente do que no grupo R. As agulhas calibre $25 \mathrm{G}$ foram mais utilizadas pelo grupo $\mathrm{R}$ (Tabela I). O grupo R não utilizou agulhas calibres $26 \mathrm{G}$ e $29 \mathrm{G}$. A qualidade das referências anatômicas (processos espinhosos) foram igualmente distribuídas entre os dois grupos (Tabela II). O número de bloqueios realizados pelos ME variou entre $31 \mathrm{e}$ 77.
As curva de aprendizado para o atributo 1 - sucesso na primeira tentativa - mostraram que $\mathrm{A}$ (21 bloqueios) e $\mathrm{E}$ (17 bloqueios) atingiram a proficiência mais precocemente do que os $B$ (44 bloqueios) e C (57 bloqueios). D (31 bloqueios) não obteve proficiência ao fim dos primeiros seis meses de residência (Tabela III e Figura 1).

Tabela III - Número de Bloqueios até Obtenção de Proficiência em Obter Sucesso na Primeira Tentativa: Comparação entre os ME

\begin{tabular}{lcc}
\hline ME & Total de bloqueios & Bloqueios até proficiência \\
\hline A & 63 & $21^{*}$ \\
B & 54 & 44 \\
C & 77 & 57 \\
D & 31 & Não atingiu \\
E & 50 & $17^{*}$ \\
\hline
\end{tabular}

${ }^{*}=p<0,05$ comparados a B e C

Tabela I - Parâmetros para Cálculo das Somas Cumulativas dos Atributos

\begin{tabular}{lccccc}
\hline & $p_{1}{ }^{1}$ & $p_{0}{ }^{2}$ & $s^{3}$ & $h_{0}, h_{1}{ }^{4}$ & $\alpha, \beta^{5}$ \\
\hline Sucesso na primeira tentativa & $35 \%$ & $50 \%$ & 2,38 & 17,88 & 0,1 \\
Sucesso no primeiro espaço & $10 \%$ & $20 \%$ & 0,20 & 3,81 & 0,1 \\
Sucesso da anestesia $\geq 95 \%$ & $5 \%$ & $20 \%$ & 0,14 & 1,80 & 0,1 \\
Sucesso da anestesia $\geq 90 \%$ & $10 \%$ & $20 \%$ & 0,20 & 3,81 & 0,1 \\
\hline
\end{tabular}

1 = taxa aceitável de insucesso; 2 = taxa inaceitável de insucesso; 3 = sigma; 4 = limites inferior e superior de controle; 5 = probabilidade de erros tipos I e II

Tabela II - Características Demográficas e das Técnicas de Punção Subaracnóidea: Comparação entre os Grupos

\begin{tabular}{|c|c|c|c|c|c|}
\hline Parâmetro & Grupo R & Grupo A & Parâmetro & Grupo R & Grupo A \\
\hline Idade (anos) * & $47,96 \pm 7,53$ & $44,74 \pm 19,02$ & $\operatorname{Sexo}(M / F)^{* *}$ & $64 / 36 \#$ & $49 / 51$ \\
\hline Acesso ** & & & Decúbito ** & & \\
\hline Mediano & 74,23 & 55,78 & Lateral & 91,15 & 69,31 \\
\hline Paramediano & $25,76 \#$ & 44,21 & Sentado & $8,84 \#$ & 30,68 \\
\hline Posicionamento ** & & & Processos Espinhosos ** & & \\
\hline Satisfatório & 85,76 & 80,30 & Palpação impossível & 4,61 & 7,87 \\
\hline \multirow[t]{2}{*}{ Insatisfatório } & 13,46 & 18,56 & Palpação difícil & 23,46 & 24,30 \\
\hline & & & Palpação fácil & 71,92 & 67,98 \\
\hline \multicolumn{6}{|l|}{ Calibre da agulha ** } \\
\hline $22 \mathrm{G}$ & 0,38 & 1,51 & & & \\
\hline $25 G$ & $44,61 \#$ & 25,75 & & & \\
\hline $26 \mathrm{G}$ & $0 \#$ & 10,22 & & & \\
\hline $27 G$ & 55 & 49,62 & & & \\
\hline $29 G$ & $0 \#$ & 12,87 & & & \\
\hline
\end{tabular}

* = média $\pm \mathrm{DP} ;{ }^{* *}=$ freqüência expressa em percentagem; \# = p <0,05 entre os grupos 


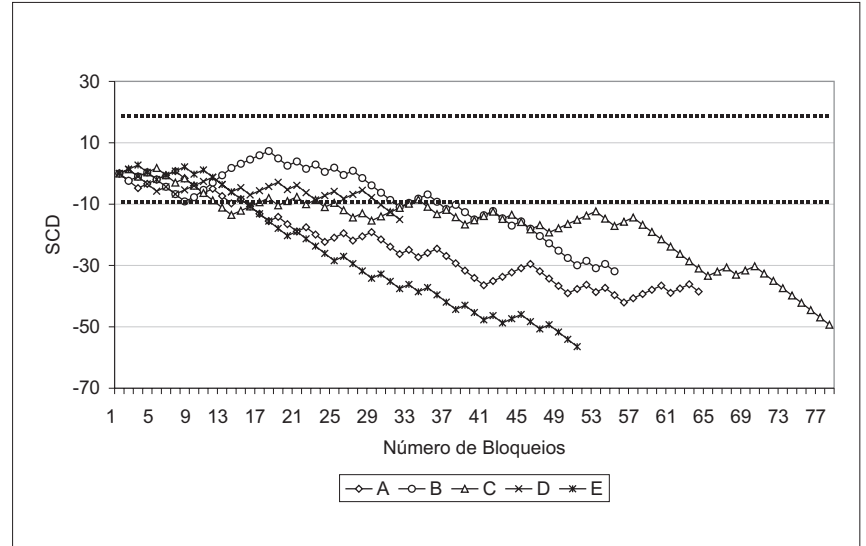

Figura 1 - Soma Cumulativa de Desvios (SCD) para o Sucesso na Primeira Tentativa de Punção Subaracnóidea: Comparação entre os ME

Acurva de aprendizado para o atributo 2 -sucesso no primeiro espaço - mostraram que $\mathrm{A}$ ( 25 bloqueios) e $\mathrm{B}$ ( 25 bloqueios) atingiram a proficiência mais precocemente do que $\mathrm{C}$ ( 61 bloqueios), enquanto $D$ (31 bloqueios) e $E$ (50 bloqueios) não atingiram a proficiência (Tabela IV e Figura 2).

Tabela IV - Número de Bloqueios até a Proficiência em se Obter Sucesso no Primeiro Espaço Inter-Espinhoso Escolhido: Comparação entre os ME

\begin{tabular}{lcc}
\hline ME & Total de bloqueios & Bloqueios até proficiência \\
\hline A & 63 & $25^{*}$ \\
B & 54 & $25^{*}$ \\
C & 77 & 61 \\
D & 31 & Não atingiu \\
E & 50 & Não atingiu \\
\hline
\end{tabular}

${ }^{*}=p<0,05$ comparados a C e E

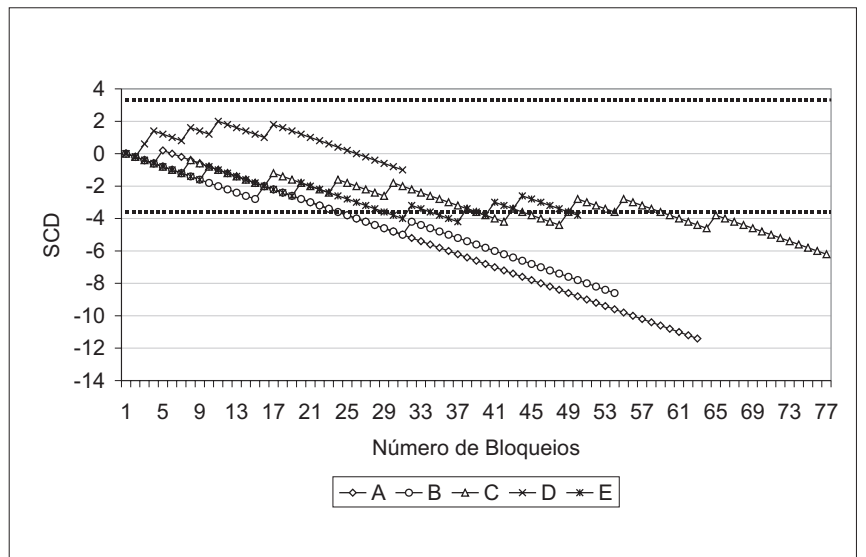

Figura 2 - Soma Cumulativa de Desvios (SCD) para o Sucesso da Punção Subaracnóidea no Primeiro Espaço Escolhido: Comparação entre os ME

Revista Brasileira de Anestesiologia

Vol. 51, № 3, Maio - Junho, 2001
As curvas de aprendizado para o atributo 3 - sucesso da anestesia cirúrgica - mostraram que apenas $C$ (49 bloqueios) e $\mathrm{E}$ (13 bloqueios) atingiram a proficiência durante os primeiros seis meses de treinamento, quando o LIC foi calculado a partir da taxa aceitável de falhas de $5 \%$ (Tabela Ve Figura 3). Quando a taxa aceitável de falhas para este atributo foi fixada em $10 \%$, todos os ME atingiram proficiência, embora com números de bloqueios significativamente diferentes (Tabela VI e Figura 4).

Tabela V - Número de Bloqueios até a Proficiência em se Obter Sucesso da Anestesia Cirúrgica $\geq 95 \%$ dos Pacientes: Comparação entre os ME

\begin{tabular}{lcc}
\hline ME & Total de bloqueios & Bloqueios até proficiência \\
\hline A & 63 & Não atingiu \\
B & 54 & Não atingiu \\
C & 77 & 49 \\
D & 31 & Não atingiu \\
E & 50 & $13^{*}$ \\
\hline
\end{tabular}

${ }^{*}=p<0,05$ comparados a C

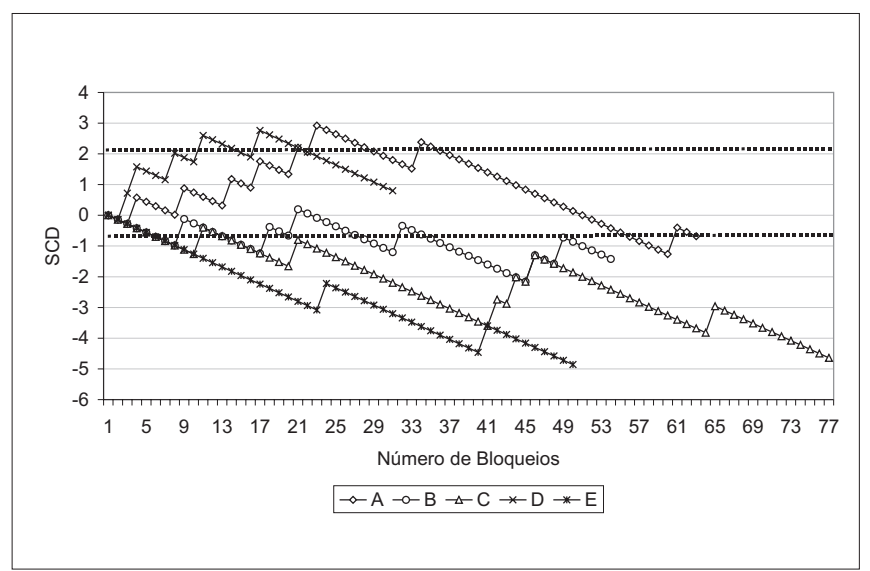

Figura 3 - Soma Cumulativa de Desvios (SCD) para o Sucesso da Anestesia $\geq 95 \%$ : Comparação entre os ME

Tabela VI - Número de Bloqueios até a Proficiência em se Obter Sucesso da Anestesia Cirúrgica em $90 \%$ ou mais dos Pacientes: Comparação entre os ME

\begin{tabular}{lcc}
\hline ME & Total de bloqueios & Bloqueios até proficiência \\
\hline A & 63 & $55^{1}$ \\
B & 54 & $35^{2}$ \\
C & 77 & $31^{3}$ \\
D & 31 & $21^{4}$ \\
E & 50 & 20 \\
\hline
\end{tabular}

$1=p<0,05$ comparados a B e C; $2=p<0,05$ comparado a $C$ e E; $3=p<$ 0,05 comparado a $D ; 4=p<0,05$ comparado a $E$ 


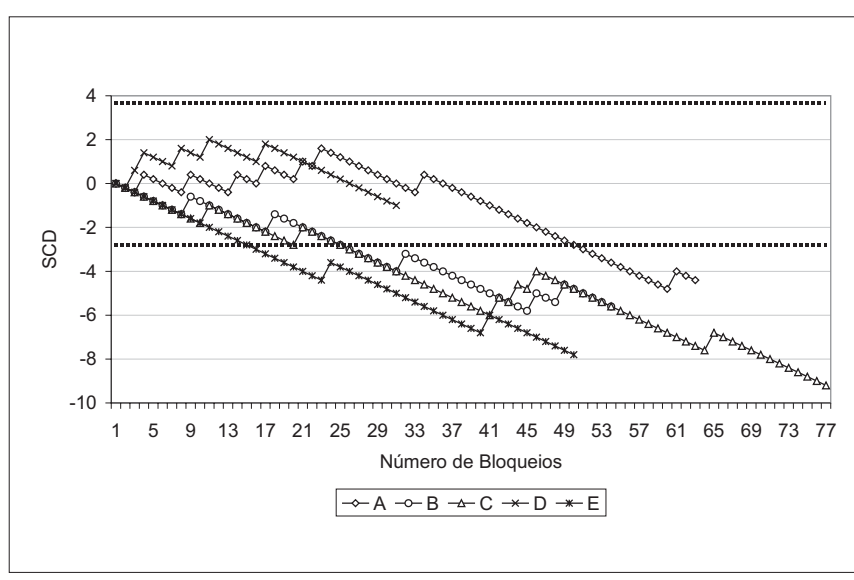

Figura 4 - Soma Cumulativa de Desvios (SCD) para o Sucesso da Anestesia $\geq 90 \%$ : Comparação entre os ME

\section{DISCUSSÃO}

Existe grande variabilidade em relação ao número de anestesias regionais a que são expostos os ME durante o treinamento $^{3,4}$. Isto interfere no grau de autoconfiança dos ME em relação à realização de bloqueios, sendo tanto mais alto quanto maior o número de procedimentos que tenham realizado $^{3}$.

Em estudo precedente, a análise das curvas de aprendizado de bloqueios subaracnóideos mostrou que o desempenho melhora significativamente em relação ao inicial após 20 bloqueios, embora a consistência - manutenção das taxas de sucesso - seja obtida somente após 45 tentativas ${ }^{5}$. Em outro estudo, a taxa de sucesso de $90 \%$ só foi atingida após 71 tentativas ${ }^{6}$.

O método da soma cumulativa dos desvios foi proposto para avaliar a proficiência de médicos em especialização na realização de procedimentos anestésicos. Utilizando este método, o número para obter proficiência em bloqueios subaracnóideos variou entre 39 e 67 tentativas ${ }^{1}$.

Nestes estudos, o sucesso foi definido como o grau de independência em relação à intervenção do instrutor para a realização da punção subaracnóidea ${ }^{1,5}$ ou a obtenção de anestesia satisfatória ${ }^{6}$.

Considerando que o método de ensino do CET prevê que durante os primeiros seis meses de treinamento os ME recebam supervisão intensiva em todos os procedimentos e ainda que nunca haviam sido estudadas curvas de aprendizado, no $C E T$, o grau de independência do $M E$ em relação às orientações do instrutor não foi utilizado como atributo de proficiência, neste estudo. Em vez dele, foram utilizados o sucesso na identificação do espaço subaracnóideo durante a primeira tentativa de punção, por representar o desempenho ideal, o sucesso da punção subaracnóidea no primeiro espaço, independentemente do número de punções da pele, como preconizado por outros autores ${ }^{7}$ e o sucesso da anestesia cirúrgica. Aanálise da soma cumulativa dos desvios foi utilizada para identificar o número de bloqueios necessários para atingir um nível de proficiência aceitável para a prática clínica.

A análise da soma cumulativa dos desvios de uma taxa aceitável de falhas é um método análogo à análise seqüencial utilizada em experimentos laboratoriais ${ }^{1,2,8}$. Os parâmetros de qualidade são chamados atributos, que definem os requisitos de conformidade de determinado item, serviço ou, neste caso, desempenho, com especificações previamente estabelecidas. O objetivo de tal análise é acompanhar graficamente o desempenho, identificando suas tendências e deslocamentos em relação ao tempo. Aanálise da SCD pode ser utilizada para comparar o desempenho de diferentes indivíduos durante o processo de aprendizado de uma técnica. Daí sua aplicação potencial ao ensino de procedimentos em Anestesiologia. Este método também pode ser utilizado por indivíduos experientes, como um instrumento de controle individual de desempenho ${ }^{2}$.

Aanálise da soma cumulativa de desvios é um procedimento simples que se inicia com a definição das taxas aceitáveis $\left(p_{1}\right)$ e inaceitáveis $\left(p_{0}\right)$ de falhas. Estas podem ser obtidas por consenso de especialistas ${ }^{1}$ ou pelo cálculo das taxas institucionais de falhas para o procedimento em estudo ${ }^{6}$. Estabelecem-se, a seguir, a magnitude aceitável dos erros tipo I $(\alpha)$ e II $(\beta)$. Com estes dados, calculam-se os limites superior e inferior de controle. Calcula-se, então, o valor de sigma (s), que representa o valor do desvio. Em um gráfico $X Y$, marcam-se no eixo Xo número dos procedimentos e, no Y, o LSC e o LIC. O gráfico inicia no ponto 0 , que representa o primeiro procedimento da série e o valor inicial da SCD. Com cada sucesso ou falha, um incremento negativo (s) ou positivo (1 - s) é adicionado, obtendo-se o escore cumulativo. ASCD declina, portanto, com cada sucesso e aumenta com cada falha. Quando a SCD diminui abaixo do LIC, a taxa de falhas observada não é considerada significativamente diferente de $p_{1}$ (a hipótese nula), com um risco de erro do tipo II igual a $\beta$. Ao contrário, quando a SCD cruza a linha que delimita o LSC a partir de baixo, então a taxa de falhas é significativamente maior do que $p_{0}$ (a hipótese alternativa), com chance de erro tipo ligual a $\alpha$. A SCD representada graficamente permite o acompanhamento do desempenho do ME durante o aprendizado de uma técnica ou procedimento. Se a SCD ultrapassar o LSC, o desempenho do ME afastou-se significativamente de $p_{0}$ e ações corretivas estão indicadas. Caso a SCD permaneça entre o LIC e o LSC, o desempenho não pode ser definido como aceitável ou inaceitável, de tal forma que mais observações precisam ser realizadas. Se a SCD mantém-se abaixo do LIC, infere-se que o ME adquiriu proficiência.

Este estudo teve por objetivo construir as curvas de aprendizado de técnicas de punção lombar e definir o número mínimo de bloqueios subaracnóideos a serem realizados para que os ME obtenham o domínio destas técnicas, durante os primeiros seis meses de treinamento.

Assim como em outros estudos ${ }^{1,2,5,6}$, estimou-se em 50 o número mínimo de procedimentos para que todos os ME obtivessem a proficiência em localizar o espaço subaracnóideo, utilizando-se como atributos o sucesso da punção na primeira tentativa ou no primeiro espaço inter-espinhoso aborda- 
do. Quando as curvas de aprendizado foram construídas baseadas na taxa de $90 \%$ de sucesso da anestesia, observou-se que 30 bloqueios foram necessários para que todos os ME atingissem a proficiência. Esta discrepância entre os números mínimos de bloqueios pode ser explicada pela alta taxa de sucesso da anestesia subaracnóidea. A variabilidade inter-individual de desempenho desta amostra reforça a necessidade de acompanhamento individualizado do desempenho dos ME, durante o aprendizado das técnicas de punção subaracnóidea ${ }^{9}$.

Neste estudo, um único aspecto do ensino do bloqueio subaracnóideo foi avaliado, ou seja, o domínio das técnicas de punção subaracnóidea. Assim, não foi estabelecido o número de bloqueios necessários para atingir a proficiência no manuseio clínico da anestesia subaracnóidea, tópico de suma relevância, para o qual outros parâmetros de acompanhamento já foram desenvolvidos ${ }^{10,11}$.

Conclui-se que a análise da soma cumulativa dos desvios é um método estatístico de fácil computação, que permite a avaliação objetiva e o acompanhamento do desempenho de médicos em especialização durante o aprendizado da anestesia subaracnóidea. Os resultados deste estudo também permitem concluir que são necessários, pelo menos 50 bloqueios, antes que seja obtida a proficiência em punções subaracnóideas.

\section{Cumulative Sum Analysis Applied to the Teaching of Spinal Anesthesia}

Getúlio Rodrigues de Oliveira Filho, M.D., Sérgio Galluf Pederneiras, M.D., Jorge Hamilton Soares Garcia, M.D.

\section{INTRODUCTION}

Spinal block learning starts by mastering lumbar puncture techniques. Teaching starts with an intensive supervision which progressively decreases as the resident improves performance, until finally it is no longer needed. The transition point between intensive supervision and no supervision must be objectively defined through quantitative performance evaluation tools, that is, through the level of proficiency.

The ideal evaluation tool should be easily computed and provide data to follow the evolution of each resident, identifying performance levels apart of acceptable standards and measuring the results of corrective interventions.

The cusum (cumulative sum) analysis (CSA) is a statistical method used to measure the proficiency of residents during the learning process of procedures such as tracheal intubation, spinal and epidural anesthesia and central venous and arterial catheterization ${ }^{1}$.

The method is easily computed and requires the calculation of three parameters: sigma deviation (s), upper control limit $(\mathrm{UCL})$, calculated as from an unacceptable failure rate $\left(p_{0}\right)$ and lower control limit (LCL), calculated as from an accepta-

Revista Brasileira de Anestesiologia

Vol. 51, № 3, Maio - Junho, 2001 ble failure rate $\left(p_{1}\right)$. To calculate CSA, one starts from a zero point, which represents the first procedure. At every success obtained in a subsequent procedure, the values is subtracted from the previous CSA and at every failure, the value of 1 - $s$ is added. So a curve representing the learning process is obtained, which goes up when there are failures and goes down when successes are achieved. When the CSA line goes beyond $U C L$, performance is considered inadequate. While the CSA line is between LCL and UCL no statistical decision may be made and additional information is needed. When the CSA line goes beyond UCL coming from above, performance is considered acceptable for not being significantly different from $p_{1}{ }^{2}$.

So, this study aimed at building learning curves for spinal puncture techniques, using the cusum analysis to determine the minimum number of procedures performed by residents to attain proficiency.

\section{METHODS}

The Medical Ethics Committee, Hospital Governador Celso Ramos, approved this study. Prospective data on the first 275 spinal blocks performed by 5 residents during the first six training months were collected (Group R). Residents were called $A, B, C, D$ and E. During the same period, a control group was formed (Group A) with a sample of 264 spinal blocks performed by 22 experienced anesthesiologists.

In Group R, blockades were performed under the supervision of instructors duly qualified by the Brazilian Society of Anesthesiology. As to lumbar puncture, residents were oriented about patients positioning, antisepsis technique, placement of sterile drapes, interspace choice, local anesthesia, needle positioning and spinal space identification by CSF observation. All blockades were performed with disposable Quincke needles. Age, gender, palpation quality of spinous processes (easy, difficult, impossible) spinal space access route (median or paramedian), position during lumbar puncture (lateral or sitting), positioning conditions (satisfactory, unsatisfactory) and lumbar puncture needle size were recorded for all patients.

The following parameters were recorded and coded as 1 if present and zero if absent:

1. Attribute 1: success on the first attempt - spinal space location with just one skin puncture.

2. Attribute 2: success on the first interspace - spinal space location in the first interspace approached although with more than one skin puncture.

3. Attribute 3 - success of anesthesia - induction of complete surgical anesthesia

To define control limits for attributes 1, 2 and 3, the following rates were derived from a sample of 264 spinal blocks performed by 22 anesthesiologists working in two hospitals during the same period (Group A). 
1. Success rate for the first attempt - number of blockades where the spinal interspace was located with just one skin puncture divided by the total number of punctures multiplied by 100 .

2. Success rate for the first space - number of blockades where spinal space was located by one or more skin punctures, but in the first interspace, divided by the total number of blockades and multiplied by 100 .

3. Success rate for anesthesia - number of blockades where the surgical procedure was performed with no need for blockade supplementation with opioids or general anesthesia, divided by the total number of blockades and multiplied by 100 .

For attribute 1, $p_{1}$ was established as 0.35 because failure rate in Group A was $35 \%$, and $p_{0}$ was arbitrarily defined as 0.5 . Similarly, $p_{0}$ was established as 0.1 and $p_{1}$ as 0.2 for attribute 2. For attribute 3 , Group A's failure rate was $5 \%$, resulting in $p_{0}$ $=0.05$ and $p_{1}$ was arbitrarily defined as 0.2 . For attribute 3 , the same success rate of $90 \%$ was adopted and defined by a consensus of specialists ${ }^{1}$, resulting in $p_{0}=0.1$ and $p_{1}=0.2$. Values of $\alpha$ (type I error) and $\beta$ (type II error) were established as 0.1 .

Variables for CSA were computed using the following formulas:

$$
\begin{aligned}
& a=\ln [(1-\beta) / \alpha] \\
& b=\ln [(1-\alpha) / \beta] \\
& P=\ln \left(p_{1} / p_{0}\right) \\
& Q=\ln \left[\left(1-p_{1}\right) /\left(1-p_{0}\right)\right] \\
& \mathrm{LCL}=-\mathrm{b} /(\mathrm{P}+\mathrm{Q}) \\
& \mathrm{UCL}=\mathrm{a} /(\mathrm{P}+\mathrm{Q}) \\
& s=\mathrm{Q} /(\mathrm{Q}+\mathrm{P})
\end{aligned}
$$

Learning curves for each attribute were calculated for each resident based on cusum analysis of deviations. Proficiency in a certain attribute (acceptance of null hypothesis) was identified by CSA values permanently below LCL. Number of blockades executed until proficiency was achieved, was compared among residents by the Chi-square and Fisher exact tests.

Patients demographic data and procedure technical characteristics were compared among groups by Student $t$ (continuous variables) or chi-square (categorical variables) tests.

\section{RESULTS}

Groups A and R did not differ as to age. Group R had more male patients. In Group A, the paramedian approach and the sitting position were more frequently used than in Group R. $25 G$ needles were more frequently used by Group R. There were no differences between groups as to 22 and $27 \mathrm{G}$ needles (Table I). Group R did not use 26 and $29 \mathrm{G}$ needles. Anato-

\begin{tabular}{|c|c|c|c|c|c|}
\hline Parameter & Group R & Group A & Parameter & Group R & Group A \\
\hline Age (years) * & $47.96 \pm 7.53$ & $44.74 \pm 19.02$ & Gender $(\mathrm{M} / \mathrm{F})^{* *}$ & $64 / 36$ \# & $49 / 51$ \\
\hline Acess ** & & & Position ** & & \\
\hline Median & 74.23 & 55.78 & Lateral & 91.15 & 69.31 \\
\hline Paramedian & 25.76 \# & 44.21 & Sitting & $8.84 \#$ & 30.68 \\
\hline Positioning ** & & & Spinal Process ** & & \\
\hline Satisfactory & 85.76 & 80.30 & Impossible palpation & 4.61 & 7.87 \\
\hline \multirow[t]{2}{*}{ Unsatisfactory } & 13.46 & 18.56 & Difficult palpation & 23.46 & 24.30 \\
\hline & & & Easy palpation & 71.92 & 67.98 \\
\hline \multicolumn{6}{|l|}{ Needle size ** } \\
\hline $22 G$ & 0.38 & 1.51 & & & \\
\hline $25 G$ & $44.61 \#$ & 25.75 & & & \\
\hline $26 G$ & $0 \#$ & 10.22 & & & \\
\hline $29 G$ & 0 \# & 12.87 & & & \\
\hline
\end{tabular}
mic references quality (spinal processes) was equally distributed among groups (Table II).

Table I - Parameters for Cumulative Sum Calculation of Attributes

\begin{tabular}{lcccc}
\hline & $p_{1}{ }^{1}$ & $p_{0}{ }^{2}$ & $s^{3}$ & $h_{0}, h_{1}{ }^{4}$ \\
\hline Success in the first attempt & $35 \%$ & $50 \%$ & 2.38 & 17.88 \\
Success in the first space & $10 \%$ & $20 \%$ & 0.20 & 3.81 \\
Anesthesia success $\geq 95 \%$ & $5 \%$ & $20 \%$ & 0.14 & 0.1 \\
Anesthesia success $\geq 90 \%$ & $10 \%$ & $20 \%$ & 0.20 & 3.80 \\
\hline
\end{tabular}

1 = acceptable failure rate; 2 = unacceptable failure rate; 3 = sigma; 4 = upper and lower control limit s; 5 = probability of type I and II errors

Table II - Demographic Data and Subarachnoid Puncture Techniques: Comparison Between Groups

${ }^{*}=$ Mean $\pm \mathrm{SD} ;{ }^{* *}=$ Frequency expressed in percentages; $\#=\mathrm{p}<0.05$ between groups 
Number of blockades performed by residents varied from 31 to 77 .

Learning curves for attribute 1 - success in the first attempt showed that $A$ ( 21 blockades) and $E$ ( 17 blockades) attained an early proficiency as compared to B (44 blockades) and C ( 57 blockades). D (31 blockades) did not attain proficiency at the end of six months (Table III and Figure 1).

Table III - Number of Blockades Needed to Attain Success in the First Attempt: Comparison Among Residents

\begin{tabular}{lcc}
\hline Residents & Total blockades & Blockades until Proficiency \\
\hline A & 63 & $21 *$ \\
B & 54 & 44 \\
C & 77 & 57 \\
D & 31 & Did not attain \\
E & 50 & $17 *$ \\
\hline
\end{tabular}

* $=p<0.05$ compared to $B$ and $C$

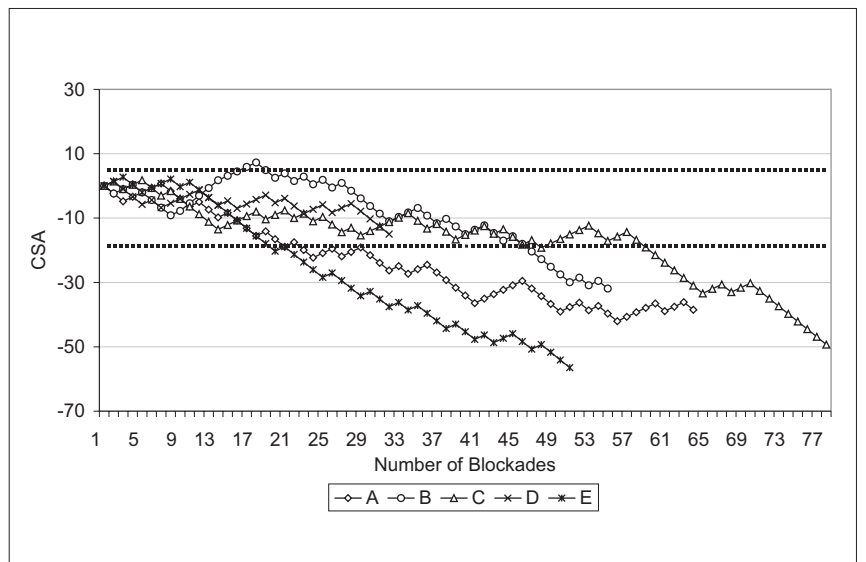

Figure 1 - Cusum Analysis of Subarachnoid Puncture Success; Comparison among Residents

Learning curves for attribute 2 - success in the first interspace - showed that $A$ ( 25 blockades) and $B$ ( 25 blockades) attained early proficiency as compared to $C$ ( 61 blockades), while $D$ (31 blockades) and $\mathrm{E}$ (50 blockades) did not attain proficiency (Table IV and Figure 2).

Table IV - Number of Blockades to Attain Success in the First Interspace Approached: Comparison Among Residents

\begin{tabular}{lcc}
\hline Residents & Total blockades & Blockades until proficiency \\
\hline A & 63 & $25^{*}$ \\
B & 54 & $25^{*}$ \\
C & 77 & 61 \\
D & 31 & Did not attain \\
E & 50 & Did not attain \\
\hline
\end{tabular}

${ }^{*}=p<0.05$ as compared to $\mathrm{C}$ and $\mathrm{E}$

Revista Brasileira de Anestesiologia

Vol. 51, № 3, Maio - Junho, 2001

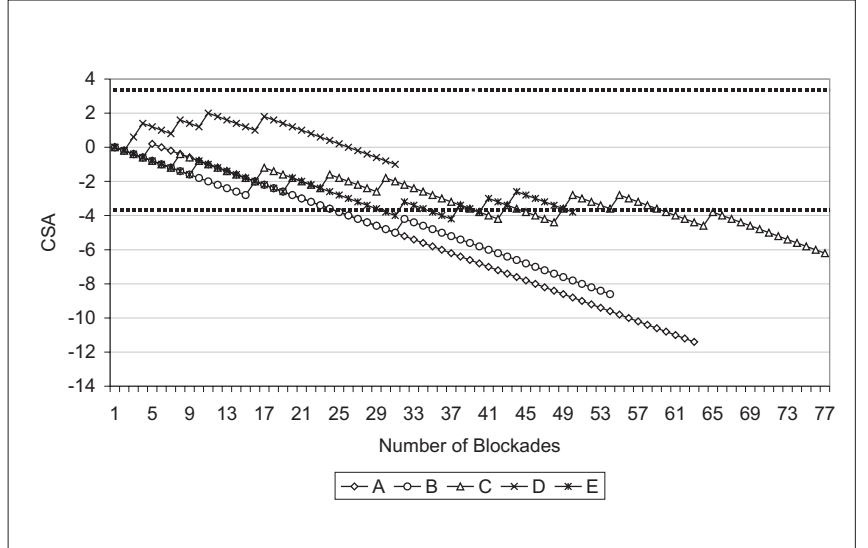

Figure 2 - Cusum Analysis of Subarachnoid Puncture Success in the First Interspace Approached; Comparison among Residents

Learning curves for attribute 3 - surgical anesthesia success showed that only $\mathrm{C}$ ( 40 blockades) and $\mathrm{E}$ ( 13 blockades) attained proficiency during the first six months, when LCL was calculated as from an acceptable failure rate of $5 \%$ (Table $V$ and Figure 3 ). When the acceptable failure rate for such attribute was increased to $10 \%$ all residents attained proficiency although with significantly different numbers of blockades (Table VI and Figure 4).

Table V - Number of Blockades to Attain Surgical Anesthesia Success in $\geq 95$ of Patients: Comparison among Residents

\begin{tabular}{lcc}
\hline Residents & Total blockades & Blockades until proficiency \\
\hline A & 63 & Did not attain \\
B & 54 & Did not attain \\
C & 77 & 49 \\
D & 31 & Did not attain \\
E & 50 & $13^{*}$ \\
\hline${ }^{*}=p<0.05$ compared to C &
\end{tabular}

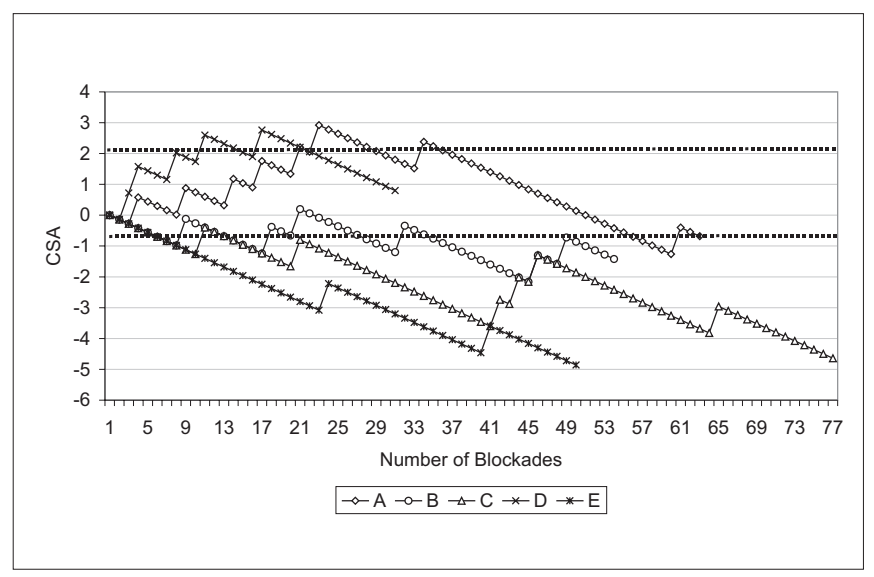

Figure 3 - Cusum Analysis of Anesthesia Success in $\geq 95 \%$ of Patients; Comparison among Residents 
Table VI - Number of Blockades to Attain Surgical Anesthesia Success in $90 \%$ of Patients or More: Comparison among Residents

\begin{tabular}{lcc}
\hline Residents & Total blockades & Blockades until proficiency \\
\hline A & 63 & $55^{1}$ \\
B & 54 & $35^{2}$ \\
C & 77 & $31^{3}$ \\
D & 31 & $21^{4}$ \\
E & 50 & 20 \\
\hline
\end{tabular}

$1=p<0.05$ as compared to $B$ and $C ; 2=p<0.05$ as compared to $C$ and $E$; $3=p<0.05$ as compared to $D ; 4=p<0.05$ as compared to $E$

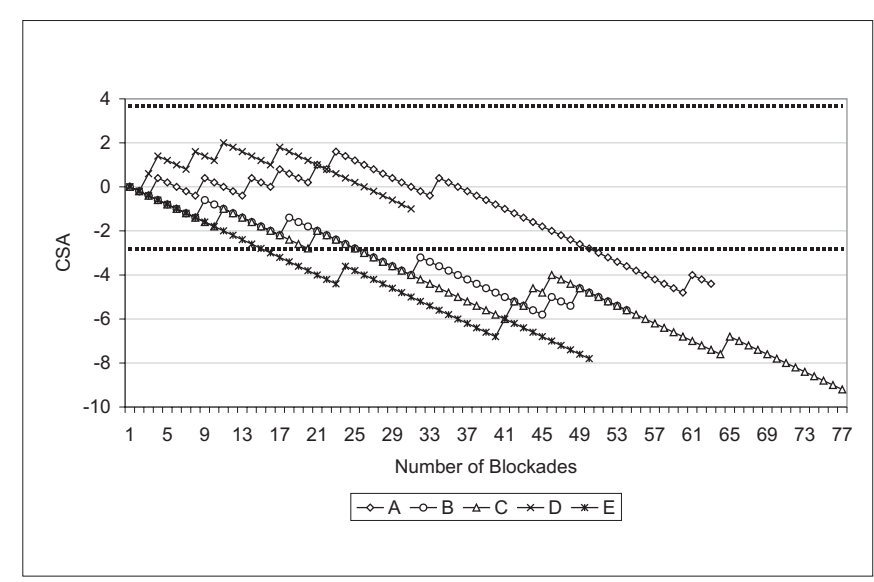

Figure 4 - Cusum Analysis of Anesthesia Success in more than $\geq 90 \%$ of Patients; Comparison among Residents

\section{DISCUSSION}

There are major variations in the number of regional anesthesias performed by residents during training ${ }^{3,4}$. This interferes with the level of self-confidence of residents with regards to blockade, which is higher when a higher number of procedures are performed ${ }^{3}$.

In a previous study, learning curve analysis of spinal blocks has shown that performance is significantly increased after 20 blockades as compared to the first one although consistency - success rate maintenance - is only obtained after 45 attempts ${ }^{5}$. In a different study, $90 \%$ success rate was only obtained after 71 attempts ${ }^{6}$. The cusum method was proposed to evaluate proficiency of physicians specializing in anesthetic procedures. With this method, the number of spinal blocks necessary for proficiency varied from 39 to 67 attempts ${ }^{1}$. In those studies, success was defined as the level of orientation independence to perform spinal blocks ${ }^{1,5}$ or achieve a satisfactory anesthesia ${ }^{6}$.

Considering that our teaching method anticipate that during the first six months of training all residents are under intensive supervision in all procedures and also that learning curves had never been studied, the level of independence of our residents was not used as a proficiency attribute. In its place, we used the success in identifying the subarachnoid space during the first puncture attempt for representing the ideal performance, the puncture success in the first interspace, regardless of the number of skin punctures, as proposed by other authors ${ }^{7}$ and the success of the surgical anesthesia. Cusum analysis was used to identify the number of blockades needed to attain an acceptable level of proficiency for clinical practice.

Cusum analysis of an acceptable failure rate is a method similar to the sequential analysis used in lab experiments ${ }^{1,2,8}$. Quality parameters are called attributes, which define the compliance requirements of a certain item, service or, in this case, with previously established specifications. The aim of such analysis is to graphically follow performance, identifying its trends and deviations as a function of time. The CSA analysis may be used to compare the performance of different people during a technique learning process, hence its potential application to the teaching of Anesthesiology procedures. This method can also be used by experienced people as a tool for individual performance control ${ }^{2}$.

The cusum analysis is a simple procedure that starts with a definition of acceptable $\left(p_{1}\right)$ and unacceptable $\left(p_{0}\right)$ failure rates. These may be obtained by specialists consensus ${ }^{1}$ or by calculating institutional failure rates for the procedure being studied $^{6}$. Then, acceptable magnitude of errors type I $(\alpha)$ and II $(\beta)$ are determined. With such data, upper and lower control limits are calculated. Then, sigma (s) value, which represents the deviation value, is calculated. In aXY chart, the number of procedures is marked in the X axle and UCL and LCL are marked in the $Y$ axle. The chart starts with 0 , which represents the first procedure and the initial CSA value. With each success or failure a negative (s) or positive (1 - s) increment is added to obtain a cumulative score. So, CSA decreases with each success and increases with each failure. When CSA goes below $L C L$, the failure rate is not considered significantly different from $p$ (null hypothesis), with a risk of error type II equals $\beta$. Conversely, when CSA crosses the line, which limits UCL coming from below, then failure rate is significantly higher than $p_{0}$ (alternative hypothesis), with a chance of error type II equals $\alpha$.

Graphically represented, CSA allows resident's performance follow up during the learning of a technique or procedure. If CSAgoes beyond UCL, the resident's performance is significantly apart from $p_{0}$ and corrective actions are indicated. If CSA remains between $L C L$ and $U C L$, the performance cannot be considered either acceptable or unacceptable and more observations are needed. If CSA remains below LCL, it is assumed that the resident has attained proficiency.

This study aimed at building learning curves for lumbar puncture learning and at defining the minimum number of spinal blocks to be performed for the technique to be mastered by the resident during the first six months of training.

Similar to other studies ${ }^{1,2,5,6}$, the minimum number of procedures for all residents to be proficient in locating the subarachnoid space was estimated in 50 , using as attributes the puncture success in the first attempt or in the first interspace approached. When learning curves were built based on a Vol. 51, № 3, Maio - Junho, 2001 
$90 \%$ anesthesia success rate, it was seen that 30 blocks were needed for all residents to become proficient. This difference in the minimum number of blockades can be explained by the spinal anesthesia success rate. The performance variation among individuals in our sample reinforces the need for a customized performance follow up during the learning of subarachnoid puncture techniques ${ }^{9}$.

In our study, only one teaching aspect of the spinal block was evaluated, that is, mastering subarachnoid puncture technique. So, it was not established the number of blockades needed to attain clinical proficiency in spinal anesthesia - an extremely relevant topic for which other follow up parameters have already been developed ${ }^{10,11}$.

The conclusion is that the cusum analysis is a statistical method easily computed which allows an objective evaluation and the performance follow up of residents during the learning process of spinal anesthesia. Our results also led to the conclusion that at least 50 blockades are needed to attain spinal anesthesia proficiency.

\section{REFERÊNCIAS - REFERENCES}

01. Kestin IG - A statistical approach to measuring the competence of anaesthetic trainees at practical procedures. $\mathrm{Br} \mathrm{J}$ Anaesth, 1995;75:805-809.

02. Williams SM, Parry BR, Schlup MMT - Quality control: an application of the cusum. Br Med J, 1992;304:1359-1361.

03. Smith MP, Sprung J, Zura A et al - A survey of exposure to regional anesthesia techniques in American anesthesia residency training programs. Reg Anesth Pain Med, 1999;24:11-16.

04. Bouaziz H, Mercier FJ, Narchi P et al - Survey of regional anesthetic practice among French residents at time of certification. Reg Anesth, 1997;22:218-222.

05. Kopacz DJ, Neal JM, Pollock JE - The regional anesthesia "learning curve". What is the minimum number of epidural and spinal blocks to reach consistency? Reg Anesth, 1996;21:182-190.

06. Konrad C, Schüpfer $\mathrm{G}$, Wietlisbach $\mathrm{M}$ et al - Learning manual skills in anesthesiology: is the recommended number of cases for anesthetic procedures? Anesth Analg, 1998;86:635-639.

07 . Sprung J, Bourke DL, Grass J et al - Predicting the difficult neuraxial block: a prospective study. Anesth Analg, 1999;89: 384-389.
08. Dixon WJ, Massey Jr FJ - Sequential Analysis, em: Dixon WJ, Massey Jr FJ - Introduction to Statistical Analysis. New York, McGraw-Hill, 1983;415-425.

09. Lawler PG, Patla VR, Garcia E et al - Assessment of training in anaesthesia and related skills. Anaesthesia, 1991;46:597.

10. Kopacz D - QA in regional anesthesia training. Quantity or quality? Reg Anesth, 1997;22:209-211.

11. Sivarajan M, Miller E, Hardy C et al - Objective evaluation of clinical performance and correlation with knowledge. Anesth Analg, 1984;63:603-607.

\section{RESUMEN}

Oliveira Filho GR, Pederneiras SG, Garcia JHS - Uso de la Suma Cumulativa de los Desvíos para Evaluación de la Proficiencia en la Enseñanza del Bloqueo Subaracnóideo

Justificativa y Objetivos - Medidas objetivas de desempeño son necesarias para la enseñanza de técnicas anestésicas. Este estudio tuvo por objetivo aplicar y describir el método de la suma cumulativa de los desvíos para la construcción de curvas de aprendizaje de la punción subaracnóidea.

Método - El suceso de la punción subaracnóidea en la primera tentativa, en el primer espacio abordado, y el suceso de la anestesia de 275 bloqueos subaracnóideos realizados por médicos en especialización (ME) durante los primeros seis meses de entrenamiento fueron utilizados para la construcción de curvas de aprendizaje, utilizando el método de las sumas cumulativas de desvíos. Las tasas aceptables de falla fueron derivadas de una muestra de 264 bloqueos subaracnóideos realizados por anestesistas. El número necesario de bloqueos para obtenerse proficiencia fue calculado, para cada ME, para cada atributo.

Resultados - Hubo una grande variabilidad en relación al número de bloqueos necesarios para alcanzar la proficiencia, dependiendo del atributo y del ME. No obstante, la mayoría de los ME alcanzó proficiencia para los atributos de suceso durante la primera tentativa o en el primero espacio interespinoso abordado, después 50 bloqueos. Una tasa de suceso de la anestesia de $90 \%$ fue obtenida por todos los ME después de 30 bloqueos.

Conclusiones - El método de la suma cumulativa de desvíos puede ser utilizado para medir objetivamente el desempeño de los médicos en especialización, durante la etapa de aprendizaje de anestesia subaracnóidea. Un número mínimo de 50 bloqueos es necesario para obtenerse proficiencia en identificar el espacio subaracnóideo. 